\title{
ON 2-DIMENSIONAL NONASPHERICAL CELL-LIKE PEANO CONTINUA: A SIMPLIFIED APPROACH
}

\author{
KATSUYA EDA, UMED H. KARIMOV, AND DUŠAN REPOVŠ
}

\begin{abstract}
We construct a functor $A C(-,-)$ from the category of path connected spaces $X$ with a base point $x$ to the category of simply connected spaces. The following are the main results of the paper: (i) If $X$ is a Peano continuum then $A C(X, x)$ is a cell-like Peano continuum; (ii) If $X$ is $n$-dimensional then $A C(X, x)$ is $(n+1)$-dimensional; and (iii) For a path connected space $X, \pi_{1}(X, x)$ is trivial if and only if $\pi_{2}(A C(X, x))$ is trivial. As a corollary, $A C\left(S^{1}, x\right)$ is a 2 -dimensional nonaspherical cell-like Peano continuum.
\end{abstract}

\section{INTRODUCTION}

Peano continua are those continua (i.e. connected metric compacta) which are also locally connected. This classical topics of topology has received renewed attention in recent years, in particular the constructions of some surprising examples which provide answers to some very interesting problems and conjectures.

In our investigations of Peano continua we constructed in [6] a new functor from the category of all path connected spaces $X$ with a base point $x$ and continuous mappings to the category of all simply connected spaces, which we named the Snake cone and denoted by $S C(-,-)$. When $X$ is a Peano continuum, $S C(X, x)$ is also a Peano continuum. We proved that in the case when $X=S^{1}$, the space $S C\left(S^{1}, x\right)$ is a noncontractible simply connected cell-like 2-dimensional Peano continuum.

In a subsequent paper [7] we showed that the space $S C\left(S^{1}, x\right)$ is even nonaspherical, i.e. $\pi_{2}\left(S C\left(S^{1}, x\right)\right) \neq 0$. Moreover, we showed that for every path connected space $X, \pi_{1}(X, x)$ is trivial if and only if $\pi_{2}(S C(X, x))$ is trivial (cf. [7] and [8]).

In the present paper we construct yet another new useful functor, called the Alternating cone, and we denote it by $A C(-,-)$. We show that while $A C(X, x)$ shares all the properties of the space $S C(X, x)$ listed above, their verification is much easier. We also show that there exists a Peano continuum $(X, x)$ such that the spaces $A C(X, x)$ and $S C(X, x)$ are not homotopy equivalent. As an example how much easier the verifications are for the new functor, we consider singular homology $H_{*}(A C(X, x))$ (cf. Theorem 3.4).

\section{Preliminaries}

We start by fixing some terminology and notations which will be used in the sequel. All undefined terms can be found in [13] or [14].

Date: June 10, 2018.

2010 Mathematics Subject Classification. Primary 54F15, 55N15; Secondary 54G20, 57M05.

Key words and phrases. Noncontractible compactum, weak homotopy equivalence, trivial shape, Peano continuum, Snake cone, Alternating cone, asphericity, cell-like space, Topologist sine curve. 
For any two points $A$ and $B$ of the plane $\mathbb{R}^{2}$, we shall denote the linear segment connecting these points by $[A, B]$. Next, by the open (resp. half-open) interval we shall mean the linear segment without its end points (resp. without one of its end points), and we shall denote it by $(A, B)$ (resp. $[A, B)$ or $(A, B])$, as usual. The unit segment of the real line $\mathbb{R}^{1}$, with the natural topology, will be denoted by $\mathbb{I}$. The point of the coordinate plane $R^{2}$ with coordinates $a$ and $b$ will be denoted by $(a ; b)$.

Consider the points $A=(0 ; 0), B=(0 ; 1), A_{n}=(1 / n ; 0), B_{n}=(1 / n ; 1)$ and let $L=[A, B], L_{2 n-1}=\left[A_{n}, B_{n}\right], L_{2 n}=\left[B_{n}, A_{n+1}\right]$ be segments in the plane $\mathbb{R}^{2}$, for $n \in \mathbb{N}=\{1,2,3, \ldots\}$. The piecewise linear Topologist sine curve $\mathcal{T}$ is a subspace of the plane $\mathbb{R}^{2}$ defined as the union of $L_{n}$ and $L$. For any topological space $X$ the cone $C(X)$ over $X$ is defined as the quotient space $C(X)=(X \times \mathbb{I}) /(X \times\{1\})$.

Suppose that $q_{i}: Z_{i} \rightarrow \mathbb{I}, i=1,2$, are mappings of topological spaces. Then the mapping $f: Z_{1} \rightarrow Z_{2}$ is said to be flat with respect to the mappings $q_{1}$ and $q_{2}$ if for every two points $a, b \in Z_{1}$ with $q_{1}(a)=q_{1}(b)$, the equality $q_{2}(f(a))=q_{2}(f(b))$ holds. Homotopy $H: Z_{1} \times \mathbb{I} \rightarrow Z_{2}$ is said to be flat if for each $t \in \mathbb{I}$ the mapping $H(-, t): Z_{1} \rightarrow Z_{2}$ is a flat mapping with respect to the mappings $q_{1}$ and $q_{2}$.

We recall the notion of the free $\sigma$-product of groups and a lemma from [4] (cf. the proof of [7, Theorem 3.1]). We only use it in the case when the index set is countable and we introduce a restricted version in the sequel. Let $\left(X_{n}, x_{n}\right)$ be pointed spaces for $n \in \mathbb{N}$ such that $X_{m} \cap X_{n}=\emptyset$, for $m \neq n$. The underlying set of the pointed space $\left(\widetilde{V}_{n \in \mathbb{N}}\left(X_{n}, x_{n}\right), x^{*}\right)$ is the union of all $X_{n}$ 's obtained by identifying all $x_{n}$ 's to a point $x^{*}$ and the topology is defined by specifying the neighborhood base as follows (cf. [1]):

(1) If $x \in X_{n} \backslash\left\{x_{n}\right\}$, then the neighborhood base of $x$ in $\widetilde{\bigvee}_{n \in \mathbb{N}}\left(X_{n}, x_{n}\right)$ is the one of $X_{n}$; and

(2) The point $x^{*}$ has a neighborhood base, each element of which is of the form: $\bigcup_{n \geq m}\left(X_{n}, x_{n}\right) \cup \bigcup_{n<m} U_{n}$ where $m \in \mathbb{N}$ and each $U_{n}$ is an open neighborhood of $x_{n}$ in $X_{n}$ for $n<m$.

Lemma 2.1. 4, Theorem A.1] Let $X_{n}$ be locally simply-connected and first countable at $x_{n}$ for each $n$. Then

$$
\pi_{1}\left(\widetilde{\bigvee}_{n \in \mathbb{N}}\left(X_{n}, x_{n}\right), x^{*}\right) \cong \mathbb{x}_{n \in \mathbb{N}} \pi_{1}\left(X_{n}, x_{n}\right)
$$

Let $G$ be a group. A commutator is an element $\left[g_{1}, g_{2}\right]=g_{1} g_{2} g_{1}^{-1} g_{2}^{-1}$ where $g_{1}, g_{2} \in G$. The normal subgroup $G^{\prime}$ of $G$ which is generated by all commutators is called the commutator subgroup of $G$.

Recall that the commutator length $\operatorname{cl}(g)$ of $g \in G^{\prime}$ is defined as the minimal number of the commutators of $G$ whose product is equal to $g$ (cf. [5]). We note that $c l(e)=0$ for the identity element $e$ of the group $G$. If $\varphi: G \rightarrow H$ is a homomorphism of a group $G$ to a group $H$, then for every $g \in G^{\prime}$,

$$
c l(\varphi(g)) \leq c l(g) .
$$

For every path connected space $\mathrm{X}$, the fundamental group $\pi_{1}(X, x)$ is independent of the choice of the base point $x$ and hence we can simply write $\pi_{1}(X)$. Finally, recall the isomorphism:

$$
H_{1}(X) \cong \pi_{1}(X) / \pi_{1}^{\prime}(X)
$$




\section{Constructions And main Results}

The Snake cone functor $S C(-,-)$ is defined as follows. For every compact space $X$ with the base point $x$, the space $S C(X, x)$ is the quotient space of the topological sum $(\mathcal{T} \times X) \bigsqcup \mathbb{I}^{2}$ via the identification of the points $(t, x) \in \mathcal{T} \times X$ with $t \in \mathcal{T} \backslash L \subset \mathbb{I}^{2}$ and the identification of each set $\{t\} \times X$ with the point $t$, for every $t \in L$ (cf. [6]).

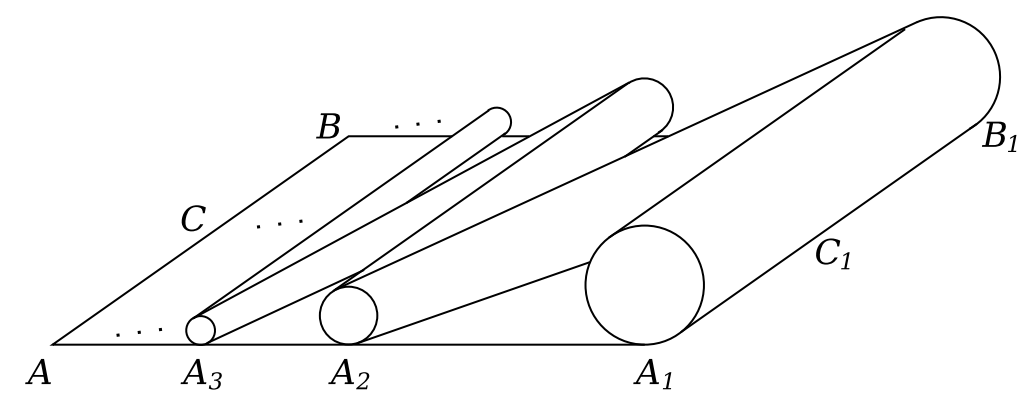

Figure 1. $S C\left(S^{1}\right)$

Let us now define the Alternating cone functor $A C(-,-)$. Let $(X, x)$ be a pointed space and let $\left(X_{n}, x_{n}\right)$ be its copies. Connect $x_{n}$ and $x_{n+1}$ by an arc for each $n \in \mathbb{N}$ and add a new point so that a neighborhood of the point contains almost all $X_{n}$ 's and almost all attached arcs. Let $Y$ be this space.

Let $A C(X, x)$ be the quotient space of $Y \times \mathbb{I}$ in which the sets $\{0\} \times X_{n}$ are identified with $\left(0, x_{n}\right)$ for all odd $n$ and the sets $\{1\} \times X_{n}$ are identified with $\left(1, x_{n}\right)$ for all even $n$.

Since $x_{n}$ and the attached arcs converge to the new point in $Y, A C(X, x)$ can be illustrated as in Figure 2. The identifited points will be denoted by $A_{2 n}=(1 / 2 n ; 0)$ and $B_{2 n-1}=(1 /(2 n-1) ; 1)$. Let $p: A C(X, x) \rightarrow \mathbb{I}^{2}$ be the natural projection and define $p(u)=\left(p_{1}(u) ; p_{2}(u)\right)$. We let $C_{n}=(1 / n ; 1 / 2)$, for $n \in \mathbb{N}$ and $C=(0 ; 1 / 2)$.

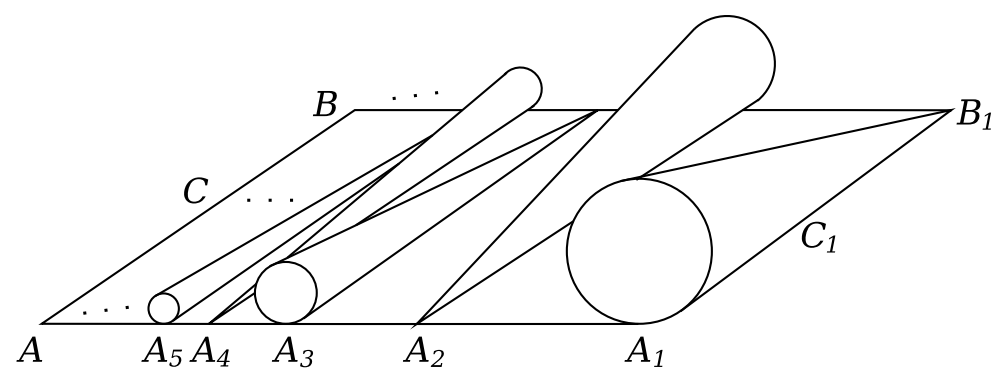

FiguRE 2. $A C\left(S^{1}\right)$

Proposition 3.1. Let $X$ be a path connected space and $x, y \in X$. Then $S C(X, x)$ and $S C(X, y)$ are homotopy equivalent. Similarly, $A C(X, x)$ and $A C(X, y)$ are also homotopy equivalent. 
Proof. We shall only prove this for $S C(X, x)$ and $S C(X, y)$, since the proof for $A C(X, x)$ and $A C(X, y)$ is similar. Let $X$ be a path connected space with $x \in X$, and $X^{*}$ the space defined in Theorem 3.4. Also, let $Y^{*}$ be the space obtained by replacing $x$ with $y$. By a deformation on $\mathbb{I}^{2}, S C(X, x)$ is homotopy equivalent to $S C\left(X^{*}, 0\right)$. Hence it suffices to establish a homotopy equivalence between $S C\left(X^{*}, 0\right)$ and $S C\left(Y^{*}, 0\right)$.

Let $f: \mathbb{I} \rightarrow X$ be a path such that $f(0)=x$ and $f(1)=y$. Define $\varphi: X^{*} \rightarrow Y^{*}$ and $\psi: Y^{*} \rightarrow X^{*}$ by:

$$
\begin{gathered}
\varphi(u)= \begin{cases}u & \text { for } u \in X \\
2 s & \text { for } s \in[0,1 / 2] \\
f(2-2 s) & \text { for } s \in[1 / 2,1]\end{cases} \\
\psi(u)= \begin{cases}u & \text { for } u \in X \\
2 s & \text { for } s \in[0,1 / 2] \\
f(2 s-1) & \text { for } s \in[1 / 2,1] .\end{cases}
\end{gathered}
$$

Then it is easy to see that $\varphi$ and $\psi$ induce a homotopy equivalence between $X^{*}$ and $Y^{*}$ which fixes 0 . This homotopy equivalence then induces a homotopy equivalence between $S C\left(X^{*}, 0\right)$ and $S C\left(Y^{*}, 0\right)$.

We are mainly interested in the homotopy types of $S C(X, x)$ and $A C(X, x)$ for the class of path connected spaces $X$. Since by Proposition 3.1 the choice of the base point $x$ does not affect the homotopy types of $S C(X, x)$ and $A C(X, x)$, we can simply write $S C(X)$ or $A C(X)$ when the choice of the base point does not matter.

Theorem 3.2. If $X$ is a continuum then $A C(X)$ is a cell-like space. If $\operatorname{dim} X=n$ then $\operatorname{dim} A C(X)=n+1$. If $X$ is a Peano continuum, then $A C(X)$ is also a Peano continuum. For any continuum $X$, the space $A C(X)$ is simply connected.

Theorem 3.3. If $X$ is a noncontractible compact space, then $A C(X)$ is also a noncontractible compact space.

Theorem 3.4. Let $X$ be a path connected space with $x \in X$ and $X^{*}$ the quotient space of the disjoint sum of the unit interval $\mathbb{I}$ and $X$, by identifying 1 of $\mathbb{I}$ and $x$. Let $\left(X_{i}, x_{i}\right)$ be copies of $\left(X^{*}, 0\right)$.

It then follows that for any natural number $n$, we have the isomorphism:

$$
H_{n+1}(A C(X)) \cong H_{n}\left(C\left(\tilde{\bigvee}_{i \in \mathbb{N}}\left(X_{2 i-1}, x_{2 i-1}\right)\right) \vee C\left(\tilde{\bigvee}_{i \in \mathbb{N}}\left(X_{2 i}, x_{2 i}\right)\right)\right)
$$

where the attaching points of the two cones are the identification points $x_{2 i-1}$ and $x_{2 i}$.

Theorem 3.5. Let $X$ be a path connected space. Then $\pi_{1}(X)$ is trivial if and only if $\pi_{2}(A C(X))$ is trivial.

It is easy to see that if $\{a, b\}$ is a two-point space with the discrete topology then the space $A C(\{a, b\})$ is path connected, whereas the space $S C(\{a, b\})$ is not. The following theorem shows their difference from another perspective:

Theorem 3.6. There exists a Peano continuum $X$ such that $A C(X)$ is not homotopy equivalent to $S C(X)$. 


\section{Proofs of Theorems 3.2 and 3.3}

In this section we shall prove Theorems 3.2 and 3.3 . The argument is similar to the one which was used for proving analogous statements concerning $S C(X)$.

Proof of Theorem 3.2. Since $X$ is a compactum, the space $A C(X)$ is the inverse limit of an inverse sequence of contractible compact metrizable spaces. Therefore $A C(X)$ is a compact metrizable space of trivial shape, i.e. cell-like.

If $\operatorname{dim} X=n$ and $X$ is compact and metrizable then the dimension of its onepoint compactification $Y$ is equal to $n$ and thus $\operatorname{dim}(Y \times \mathbb{I})=n+1$. Therefore $\operatorname{dim} A C(X)=n+1$. If $X$ is a Peano continuum then $A C(X)$ is compact, metrizable and locally path connected, hence also a Peano continuum.

Let us prove that $A C(X)$ is a simply connected space. Let $U=p_{2}^{-1}([0,1))$ and $V=p_{2}^{-1}((0,1])$ (cf. Figure 1). The sets $U$ and $V$ are homotopy equivalent to $p_{2}^{-1}(\{0\})$ and $p_{2}^{-1}(\{1\})$, respectively. Their intersection $U \cap V$ is homotopy equivalent to $p_{2}^{-1}(\{1 / 2\})$. Obviously, the mapping

$$
\pi_{1}\left(p_{2}^{-1}(\{1 / 2\})\right) \rightarrow \pi_{1}\left(p_{2}^{-1}(\{0\})\right) * \pi_{1}\left(p_{2}^{-1}(\{1\})\right)
$$

is surjective and the intersection $U \cap V$ is path connected. Therefore by the van Kampen theorem, the union $U \cup V=A C(X)$ is simply connected.

For a noncontractible space $X$, the noncontractibility of $A C(X)$ can be proved similarly as for $S C(X)$ (cf. [6, Theorem 1.2]). Hence we only indicate the difference and we state the necessary lemmas.

Let $M_{y}=p_{2}^{-1}(\{y\})$ and $A C_{n}(X)=p^{-1}\left(\left[A_{2 n}, B_{2 n}\right] \cup\left[A_{2 n+1}, B_{2 n+1}\right]\right)$ be the subspaces of $A C(X)$. The following lemma can be proved analogously as 6 , Lemma $4.2]$.

Lemma 4.1. Let $n \in \mathbb{N}$ and let $H: A C_{n}(X) \times \mathbb{I} \rightarrow A C(X)$ be a mapping such that for every $y \in \mathbb{I}$ and $t \in \mathbb{I}$, the closure of the set $p_{2}\left(H\left(M_{y} \cap A C_{n}(X), t\right)\right)$ does not contain both points 0 and 1 , and such that both mappings $H(-, 0)$ and $H(-, 1)$ are flat. Then there exists a flat homotopy from $H(-, 0)$ to $H(-, 1)$.

For $s \in(0,1)$ and $t \in \mathbb{I}$, we define a property $P(s, t)$ of the flat homotopy $H$ as follows:

$H\left(M_{s} \cap A C_{n}(X), t\right) \subseteq p_{2}^{-1}((0,1))$ and the restriction of $H(-, t)$ to

$M_{s} \cap A C_{n}(X)$ is homotopic to the identity mapping on $M_{s} \cap A C_{n}(X)$ in $p_{2}^{-1}((0,1))$.

We remark that by the flatness of $H$, if $H\left(M_{s} \cap A C_{n}(X), t\right) \subseteq p_{2}^{-1}((0,1))$, then there is a neighborhood $U$ of $(s ; t)$ such that $H\left(M_{s^{\prime}} \cap A C_{n}(X), t^{\prime}\right) \subseteq p_{2}^{-1}((0,1))$ for any $\left(s^{\prime} ; t^{\prime}\right) \in U$. The following lemma can be proved similarly as [6, Lemma 4.3].

Lemma 4.2. Let $X$ be a noncontractible space and $H: A C_{n}(X) \times \mathbb{I} \rightarrow A C(X)$ a flat homotopy. If $H\left(M_{0} \cap A C_{n}(X), t_{0}\right) \subseteq p_{2}^{-1}((0,1))$, then there exists a neighborhood $U$ of $\left(0 ; t_{0}\right)$ such that $H$ does not satisfy $P(s, t)$ for any $(s ; t) \in U$ with $s>0$. An analogous statement holds for $H\left(M_{1} \cap A C_{n}(X), t_{0}\right) \subseteq p_{2}^{-1}((0,1))$.

By Lemmas 4.1] and 4.2, we can use the proof of [6, Lemma 4.4] to verify also the next lemma.

Lemma 4.3. Let $X$ be a noncontractible space. If $H: A C_{n}(X) \times \mathbb{I} \rightarrow A C(X)$ is a flat homotopy such that $H(u, 0)=u$ for every $u \in A C_{n}(X)$, then $H(-, 1)$ is not a constant mapping. 
We can now regard the proof of [6. Theorem 1.2] also as a proof of Theorem 3.3 . We shall only retrace its line of argument.

Proof of Theorem 3.3. Suppose that the space $A C(X)$ is contractible. Then there exists a homotopy $H: A C(X) \times T \rightarrow A C(X)$ connecting the identity mapping with the constant one. By compactness of the time interval $\mathbb{I}$, for every $a=(0 ; y) \in$ $\{0\} \times \mathbb{I}$, there exists $\varepsilon_{0}>0$ such that the diameters of $p_{2} \circ H\left(O_{\varepsilon_{0}}(a), t\right)$ are less than 1 for all $t \in \mathbb{I}$. Hence, by compactness of $[A, B]$, there exists $\varepsilon_{1}>0$ such that the diameters $p_{2} \circ H\left(O_{\varepsilon_{1}}(a), t\right)$ are less than 1 for all $a=(0 ; y) \in\{0\} \times \mathbb{I}$ and all $t \in \mathbb{I}$. Let $n$ be a number such that $1 / n<\varepsilon_{1}$. By Lemma 4.1 we may then assume that $\left.H\right|_{A C_{n}(X) \times \mathbb{I}}$ is a flat contraction. However, this contradicts Lemma 4.3 ,

\section{Proofs of Theorems 3.4 and 3.5}

In this section we prove Theorems 3.4 and 3.5 . Here we shall see a very different feature of $A C(X)$ in comparison with $S C(X)$.

Let $U=p_{2}^{-1}([0,1))$ and $V=p_{2}^{-1}((0,1])$. The spaces $U, V$ and $U \cap V$ are homotopy equivalent to $p_{2}^{-1}(\{0\}), p_{2}^{-1}(\{1\})$ and $p_{2}^{-1}(\{1 / 2\})$, respectively. Let $i_{U}$ and $i_{V}$ be the inclusion maps from $U \cap V$ to $U$ and $V$, respectively. Consider the following part of the Mayer-Vietoris exact sequence for the singular homology:

$$
\begin{gathered}
H_{n+1}(U) \oplus H_{n+1}(U) \longrightarrow H_{n+1}(A C(X)) \longrightarrow H_{n}(U \cap V) \stackrel{\left(i_{\left.U_{*}, i_{V^{*}}\right)}\right.}{\longrightarrow} \\
H_{n}(U) \oplus H_{n}(V) \longrightarrow H_{n}(A C(X)) .
\end{gathered}
$$

Since the inclusion maps from $U$ to $A C(X)$ and from $V$ to $A C(X)$ are inessential, we have the following exact sequence:

$$
0 \longrightarrow H_{n+1}(A C(X)) \longrightarrow H_{n}(U \cap V) \stackrel{\left(i_{U_{*}}, i_{V *}\right)}{\longrightarrow} H_{n}(U) \oplus H_{n}(V) \longrightarrow 0 .
$$

We recall the following fact:

Lemma 5.1. 9, Theorem 1.3] Let $C X$ be the cone over a space $X$ and $C X \vee C Y$ the one-point union with two points of the base spaces $X$ and $Y$ being identified to a point. Then, the following holds for $n \geq 1$ :

$$
\mathrm{H}_{n}(X \vee Y) \cong \mathrm{H}_{n}(X) \oplus \mathrm{H}_{n}(Y) \oplus \mathrm{H}_{n}(C X \vee C Y) .
$$

Proof of Theorem 3.4. Let $r_{X}: X \vee Y \rightarrow X$ and $r_{Y}: X \vee Y \rightarrow Y$ be the retractions from Lemma [5.1. Then $\mathrm{H}_{n}(C X \vee C Y)$ is the kernel of the homomorphism $\left(\left(r_{X}\right)_{*},\left(r_{Y}\right)_{*}\right): \mathrm{H}_{n}(X \vee Y) \rightarrow \mathrm{H}_{n}(X) \oplus \mathrm{H}_{n}(Y)$.

$$
\begin{aligned}
& \begin{array}{llc}
V & \cong p_{2}^{-1}(\{1\})= & p_{2}^{-1}(\{1\}) \\
\uparrow i_{V} & \uparrow r_{B}
\end{array} \\
& U \cap V \cong p_{2}^{-1}(\{1 / 2\}) \cong\left(p_{2}^{-1}(\{0\}), A\right) \vee\left(p_{2}^{-1}(\{1\}), B\right) \\
& \downarrow i_{U} \simeq \quad \downarrow r_{A} \\
& U \cong p_{2}^{-1}(\{0\})=p_{2}^{-1}(\{0\})
\end{aligned}
$$

Since the diagram above is homotopy commutative, $H_{n+1}(A C(X))$ is isomorphic to $H_{n}\left(C\left(p_{2}^{-1}(\{0\})\right) \vee C\left(p_{2}^{-1}(\{1\})\right)\right.$.

For each $n$, let $X_{n}$ be a copy of the one-point union of $X$ and the unit interval $\mathbb{I}$, where the attaching point is $0 \in \mathbb{I}$. Let $x_{n}$ be a copy of $1 \in \mathbb{I}$. We regard $X_{n}$ as $\left[C, C_{n}\right] \cup p^{-1}\left(\left\{C_{n}\right\}\right)$. Then $p_{2}^{-1}(\{0\})$ is homotopy equivalent to $\widetilde{\bigvee}_{n \in \mathbb{N}}\left(X_{2 n-1}, x_{2 n-1}\right)$, 
$p_{2}^{-1}(\{1\})$ is homotopy equivalent to $\widetilde{V}_{n \in \mathbb{N}}\left(X_{2 n}, x_{2 n}\right)$ and $p_{2}^{-1}(\{1 / 2\})$ is homotopy equivalent to $\widetilde{\bigvee}_{n \in \mathbb{N}}\left(X_{n}, x_{n}\right)$. This verifies the assertion.

Proof of Theorem 3.5. By the result preceding the proof of Theorem 3.4 we have the exact sequence:

$$
0 \longrightarrow H_{2}(A C(X)) \longrightarrow H_{1}(U \cap V) \stackrel{\left(i_{U *}, i_{V^{*}}\right)}{\longrightarrow} H_{1}(U) \oplus H_{1}(V) \longrightarrow 0 .
$$

Suppose that $\pi_{1}(X)$ is trivial. Since $\pi_{1}(U \cap V)$ is isomorphic to $\mathbb{x}_{n \in \mathbb{N}} \pi_{1}\left(X_{n}, x_{n}\right)$, $\pi_{1}(U \cap V)$ is also trivial. Hence $H_{1}(U \cap V)$ is trivial, which implies that $H_{2}(A C(X))$ is trivial, by the above exact sequence.

On the contrary, suppose that $\pi_{1}(X)$ is nontrivial. Choose a non-trivial element $a$ of $\pi_{1}(X)$ and let $a_{n} \in \pi_{1}\left(X_{n}\right)$ be copies of $a$. Then there exists an element $a^{*}$ of the group $\pi_{1}(U \cap V)$ such that its image under the induced homomorphism from retraction of the space $U \cap V$ to the $\left[C, C_{2 n}\right] \cup p^{-1}\left(\left[C_{2 n}, C_{1}\right]\right)$ is

$$
\left[a_{1}, a_{2}\right]\left[a_{3}, a_{4}\right] \cdots\left[a_{2 n-1}, a_{2 n}\right] .
$$

By [10, Theorem 1], the commutator length of this element is $n$. Since the number $n$ is arbitrary, $a^{*}$ does not belong to the commutator subgroup of $\pi_{1}(U \cap V)$. Therefore the homology class $\left[a^{*}\right] \in H_{1}(U \cap V)$ is nontrivial (2). However, its images $i_{U *}\left(\left[a^{*}\right]\right)$ and $i_{V *}\left(\left[a^{*}\right]\right)$ in $H_{1}(U)$ and $H_{1}(V)$, respectively, are obviously trivial. By the exactness of the Mayer-Vietoris sequence, the group $H_{2}(A C(X))$ is nontrivial. Since by Theorem 3.2, $\pi_{1}(A C(X))$ is trivial, it follows by the Hurewicz Theorem that $\pi_{2}(A C(X))$ is isomorphic to $H_{2}(A C(X))$ and hence is also nontrivial.

Proof of Theorem 3.6. Let $\mathbb{H}$ be the well-known Hawaiian earring, which is obviously a Peano continuum. Let us show that $S C(\mathbb{H})$ is not homotopy equivalent to $A C(\mathbb{H})$. Consider the embedding $\varphi: \mathbb{H} \rightarrow S C(\mathbb{H})$, defined by $\varphi(x)=\left(A_{1}, x\right) \in S C(\mathbb{H})$ in Figure 1, In the sequel we shall identify $\mathbb{H}$ with its image $\varphi(\mathbb{H})$.

We show that the mapping $\varphi$ is not homotopy equivalent to the constant one. To show this by contradiction, suppose that this is not the case and that there exists a homotopy $H: X \times I \rightarrow S C(\mathbb{H})$ such that $H(x, 0)=\varphi(x)$ and $H(-, 1)$ is a constant mapping to some point $*$.

Since $S C(\mathbb{H})$ is a path connected space, we may assume without loss of generality, that $* \in[A, B] \subset \mathcal{T}$. Let $a=\inf \left\{t: H\left(x^{*}, t\right) \in[A, B]\right\}$. The image $H\left(x^{*} \times[0, a]\right)$ is a Peano continuum such that $H\left(x^{*}, 0\right)=\left(A_{1}, x^{*}\right) \in \mathcal{T} \subset S C(X), H\left(x^{*}, 1\right) \in$ $[A, B] \subset \mathcal{T} \subset S C(X)$. Since $[A, B]$ is a path connected component of the space $\mathcal{T}$, the set $p \circ H\left(x^{*} \times[0, a]\right)$ is not a subset of $\mathcal{T}$ and there exists $b \in[0, a]$ for which $p \circ H\left(x^{*}, b\right) \notin \mathcal{T}$ and consequently $H\left(x^{*}, b\right) \in \mathbb{I}^{2} \backslash \mathcal{T}$.

Since $b<a$, we have $p \circ H\left(x^{*},[0, b]\right) \cap[A, B]=\emptyset$ and so $H\left(x^{*},[0, b]\right) \cap[A, B]=\emptyset$. There exists a neighborhood $D$ of $H\left(x^{*}, b\right)$ which is a disk and is disjoint from $\mathcal{T}$. We have a neighborhood $W$ of $x^{*}$ for which $H(W \times[0, b]) \subset(S C(X) \backslash[A, B])$ and $H(W \times b) \subset D$. Since the disk $D$ is contractible and the space $\mathbb{H}$ is a retract of $S C(X) \backslash[A, B]$, we get a homotopy of $W$ to $\mathbb{H}$ connecting the embedding with a constant mapping. However, the embedding of $W$ to $\mathbb{H}$ is essential, which is a contradiction. Now we conclude that $\varphi$ is an essential mapping.

Next we show that every mapping $\psi: \mathbb{H} \rightarrow A C(\mathbb{H})$ is inessential. First, observe that the embeddings of $U$ and $V$ to $A C(\mathbb{H})$ are inessential. For instance, for $U$, we have a deformation retraction of $U$ to $p_{y}^{-1}(A)$ which can be contracted in $F(\mathbb{H})$ to a point. We have $\psi\left(x^{*}\right) \in U$ or $\psi\left(x^{*}\right) \in V$ and we only deal with the case $\psi\left(x^{*}\right) \in U$. 
There exists a neighborhood $\mathcal{O}$ of $x^{*}$ such that $\psi(\mathcal{O}) \subset U$. All but finitely many circles of $\mathbb{H}$ are contained in $\mathcal{O}$. Since $A C(X)$ is simply connected by Theorem 3.2 the restrictions of $\psi$ to the finitely many circles are inessential mappings. Hence, as a total, $\psi$ is an inessential map.

Suppose now that the spaces $S C(X)$ and $A C(X)$ are homotopy equivalent. Then there exist mappings $f: S C(X) \rightarrow A C(X)$ and $g: A C(X) \rightarrow S C(X)$ such that the composition $g f$ is homotopic to the identity mapping. Thus $\varphi \simeq g f \varphi$. However, $g f \varphi$ is an inessential mapping, because $f \varphi$ is inessential and $\varphi$ was shown to be an essential mapping. Contradiction.

In view of Theorems $3.2,3.3$, and 3.4 it is natural to ask the following question (cf. [7]):

Problem 5.2. Does there exist a finite-dimensional noncontractible Peano continuum all homotopy groups of which are trivial?

\section{Acknowledgements}

This research was supported by the Slovenian Research Agency grants P1-02920101, J1-9643-0101, J1-2057-0101 and J1-4144-0101. The first author was also supported by the Grant-in-Aid for Scientific Research (C) of Japan No. 20540097 and 23540110. We thank the referee for comments and suggestions.

\section{REFERENCES}

[1] M. G. Barrat, J. Milnor, An example of anomalous singular homology, Proc. Amer. Math. Soc. 13 (1962), 293-297.

[2] K. Borsuk, Theory of Shape, Monografie Math. 59, PWN, Warsaw, 1975.

[3] G. E. Bredon, Sheaf Theory, Second Ed., Graduate Texts in Math. 170, Springer, New York, 1997.

[4] K. Eda, Free $\sigma$-products and noncommutatively slender groups, J. Algebra 148 (1992), 243263.

[5] K. Eda, U. H. Karimov, D. Repovš, On (co)homology locally connected spaces, Topology Appl. 120 (2002), 397-401.

[6] K. Eda, U. H. Karimov, D. Repovš, A construction of simply connected noncontractible cell-like two-dimensional continua, Fund. Math. 195 (2007), 193-203.

[7] K. Eda, U. H. Karimov, D. Repovš, A nonaspherical cell-like 2-dimensional simply connected continuum and related constructions, Topology Appl. 156 (2009), 515-521.

[8] K. Eda, U. H. Karimov, D. Repovš, On the second homotopy group of $S C(Z)$, Glasnik Math. 44(64):2 (2009), 493-498.

[9] K. Eda and K. Kawamura, Homotopy groups and homology groups of the $n$-dimensional Hawaiian earring, Fund. Math. 165 (2000), 17-28.

[10] H. B. Griffiths, A note on commutators in free products. II, Proc. Cambridge Philos. Soc. 51 (1955), 245-251.

[11] U. H. Karimov, D. Repovš, On suspensions of contractible compacta of trivial shape, Proc. Amer. Math. Soc. 127:2 (1999), 627-632.

[12] R. C. Lyndon, P. E. Schupp, Combinatorial Group Theory, Princeton Univ. Press, Princeton, N.J., 1971.

[13] E. H. Spanier, Algebraic Topology, Springer-Verlag, New York, Heidelberg, Berlin, 1966.

[14] G. W. Whitehead, Elements of Homotopy Theory, Springer-Verlag, Berlin, 1978. 
School of Science and Engineering, Waseda University, Tokyo 169-8555, Japan

E-mail address: eda@logic.info.waseda.ac.jp

Institute of Mathematics, Academy of Sciences of Tajikistan, Ul. Ainy $299^{A}$, Dushanbe 734063, TAJIKISTAN

E-mail address: umedkarimov@gmail.com

Faculty of Mathematics and Physics, and Faculty of Education, University of LjublJana, P.O.Box 2964, LuUbluana 1001, Slovenia

E-mail address: dusan.repovs@guest.arnes.si 